\title{
With the cooler weather, risk of influenza increases
}

\author{
Volume 2 Issue 6 - 2015 \\ Susann Kircher \\ Alabama Society of Allergy, Asthma \& Immunology, University of \\ South Alabama, USA \\ Correspondence: Susann Kircher, Alabama Society of \\ Allergy, Asthma \& Immunology, University of South Alabama, \\ Birmingham, Alabama Area, USA, Email susannk70@yahoo.com
}

Keywords: influenza, guidelines, virus, flu, allergy

As the weather cools and the leaves change color, those in the medical field are aware that these seasonal changes also herald infuenza (flu) season. The flu virus mutates every year at a rate that is faster and in some ways more advanced than most viruses. This is why it is hard to "predict" which strains of the virus should be placed in the vaccine from year to year. The flu does not descriminate, with everyone being at risk. However, we know that those at especially high risk are the very young, the elderly, pregnant women, the immunocompromised, and those with asthma.

In the United States, Flu season typically is from October to late April/early May. Influenza often presents with fever, chills, cough, lethargy, rhinorrhea, sore throat and muscle aches. In those at increased risk, such as immunocompromised patients and patients with asthma, flu can lead to secondary bacterial pneumonia, sepsis and dehydration. In the United states, several thousand people die from the flu every year.

There are several types of flu vaccines on the market. The most common is an inactivated (dead) trivalent vaccine, which contains two " $A$ " strains and one "B" strain of influenza virus. The Northern Hemisphere seasonal vaccine for 2015 includes the following strains:

1. an A/ California/7/2009(H1N1)pdm09-like virus;

2. an A/Switzerland/9715293/2013 (H3N2)-like virus; and

3. an $\mathrm{B} /$ Phuket/3073/2013-like virus

The newer quadrivalent vaccines contain the above vaccines as well as a B/Brisbane/60/2008-like virus.

Both the trivalent and quadrivalent vaccines are given intramuscularly. There is also an intranasal flu vaccine, which this year will be quadrivalent. The intranasal vaccine is a live attenuated vaccine and should only be administered to patients without any underlying medical conditions, such as asthma or immune suppression. ${ }^{1}$ There are special considerations regarding administration of the flu vaccine.

There is a question as to whether or not a patient with egg allergy can receive the flu vaccine. The latest Practice Parameters by the Joint Task Force from the American College of Allergy, Asthma, and Immunology and the Ammerican Academy of Allergy, Asthma, and Immunology published in 2012, states that it is safe for patients with egg allergy to receive the flu vaccine. However, as I will mention below, the guidelines for 2015-2016 have been revised regarding egg allergy. ${ }^{2}$

There are two egg free vaccines on the market approved for patients 18years and older. The first is called Flucelvax, prepared via cell culture, specifically the Madin Darby Canine Kidney cells. ${ }^{3}$ The other is called Flublok. ${ }^{4}$ This vaccine is prepared from hemagglutinin recombinant proteins (HA) produced from insect cells. ${ }^{2}$ The HA proteins are needed for immunity. These HA proteins are put in cells

and the cells grown in large quantities in steel containers. The HA proteins are then purified and put into Flublok. ${ }^{4}$ Technically, only Flublok is considered to be completely egg-free, as Flucelvax could contain less than $5 \times 10^{\wedge} 8 \mathrm{mg} / \mathrm{mL}$ per dose, of which ovalbumin is a miniscule amount. ${ }^{1,3,4}$

Since the 2012 guielines were published, there has been some new data and recommendations. The Advisory Committee on immunization Practices (ACIP) has made the following changes to the guidelines, based on new data:

1. Flublok is now recommended for patients age 18 or greater (previously was recommnened for those 18-49).

2. "Children 6months - 8years of age should receive 2 doses of the flu vaccine, four weeks apart", if this is the first time they are receiving the vaccine.

3. If they have received 2 doses previously, then 1 dose is sufficient. ${ }^{1}$ For the 2015-2016 flu season, the ACIP states Live Attenuated Influenza Vaccine (LAIV) is not more effective than the Inactivated Influenza Vacccine (IIV).

Therefore, either of these 2 vaccines is ok for persons age 2-49 to receive. ${ }^{1}$ Those who should not receive the LAIV include the following:

1. Persons less than 2 years old or older than 49 years old

2. Children taking aspirin

3. Anyone who has had a severe allergic reaction to LAIV

4. Pregnant women

5. Immunocompromised people

6. Patients with asthma 


\section{Patients with a history of egg allergy}

8. Patients which have been on flu antivirals in the previous 48 hours

9. Caregivers of immunocompromised patients. ${ }^{1}$

The ACIP also updated their guidelines for influenza vaccine in egg allergic patients as follows: Patients with egg allergy that presents with hives only should receive the flu vaccine.

The LAIV should not be used in these patients. These patients should receive the vaccine from a healthcare provider who is aware of the manifestations of egg allergy, such as an allergist, and the patient should be observd for 30 minutes. ${ }^{1}$ Patients with a history of egg allergy manifests as anaphylaxis should receive Flublok (if they are at least 18years of age) in the presence of an anaphylaxis specialist such as an allergist. If the patient is not old enough to receive Flublok, then IIV may be administered by an allergy specialist or another doctor able to manage and treat anaphylaxis. Again, the patient should be monitored for at least 30minutes. Persons with suspected but not confirmed egg allergy should receive Flublok if over 18 years of age. Patients who have had a severe allergic reaction to the flu vaccine, regardless of the cause, should not receive the flu vaccine again. ${ }^{5}$

In conclusion, the flu vaccine saves lives. Efficay of the flu vaccine can be as high as $70 \%$ protective. Because the flu vaccine mutates so quickly, it is recommneded to get a flu vacine yearly, usually in October. It takes about 2 weeks, after the vaccine is administered, for a healthy person to develop protective antibodies to the flu. A person is contagious and is shedding and spreading the virus 24 hours before they start showing signs and symptoms of the flu. This is why it is so important to get vaccinated, espcially for those in the healthcare industry. If you do contract the flu, antivirals such as Tamiflu can be effective if started within 48 hours of the onset of symptoms.

\section{Acknowledgements}

None.

\section{Conflicts of interest}

Author declares there are no conflicts of interest.

\section{Funding}

None.

\section{References}

1. www.cdc.gov/flu

2. Kelso JM, Greenhawt MJ, Li JT, et al. Adverse Reactions to Vaccines Practice Parameter 2012 Update. J Allergy Clin Immunol . 2012;130(1):25-43.

3. Flucelvax (Novartis) Package insert. 2012.

4. Flublok (Protein Science) Package Insert. 2013.

5. CDC. General Recommendationson immunization-recommendations of the Advispry Committee on Immunization Practices (ACIP). MMWR Recomm Rep. 2011;60(2):1-64. 